\title{
TRANSPLANTATION \\ Good long-term outcomes after pediatric en bloc transplantation
}

The lack of donor organs available for patients with end-stage renal disease has led to the use of expanded criteria kidney donors. A retrospective analysis comparing long-term outcomes following en bloc pediatric kidney transplantation (EBKT), living donor kidney transplantation (LDKT) and standard criteria deceased donor kidney transplantation (SDKT) in adult recipients shows that EBKT is a viable strategy to help address the organ deficit.

\section{We demonstrated that} excellent outcomes are possible after EBKT from donors weighing $\leq 10 \mathrm{~kg} \ldots 77$

Technical difficulties and complications have led to a reluctance to perform EBKT, especially when the donor weighs $<10 \mathrm{~kg}$. However, evidence has shown that EBKT has better outcomes than adult SDKT. "At the Hume-Lee Transplant Center, we have been performing these technically challenging transplantations for the past 25-30 years to meet the shortage of adult kidneys from deceased donors," says Amit Sharma, an author on the recent paper. "Numerous reports have compared the outcomes of EBKT with SDKT. Ours is only the second report comparing the outcomes of these pediatric kidneys with LDKT."

In their single-center study, Sharma et al. compared adult recipients of pediatric en bloc kidneys $(n=20)$ with a matched group who underwent SDKT $(n=249)$ or LDKT $(n=215)$. The outcomes from donors weighing $\leq 10 \mathrm{~kg}$ were also compared with those from donors weighing $11-15 \mathrm{~kg}$. No significant differences were found in 5-year graft and patient survival between the three groups, although allograft survival was significantly better after LDKT than after SDKT. Complications were similar in the three groups, but the incidence of acute rejection was higher in the EBKT group than in the LDKT group (10\% versus $1 \%$ ). Graft and patient survival after EBKT did not differ between the group receiving kidneys from donors weighing $\leq 10 \mathrm{~kg}$ and those receiving kidneys from donors weighing $11-15 \mathrm{~kg}$.

"We demonstrated that excellent outcomes are possible after EBKT from donors weighing $\leq 10 \mathrm{~kg}$ without a significant increase in the risk of vascular or urological complications," says Sharma. "However, good results with this technique require excellent patient selection and meticulous attention to surgical detail." Certain recipient conditions, such as diabetes mellitus, coronary artery disease and panel reactive antibody levels $>15 \%$, are considered as high risk factors with EBKT. The authors also suggest that EBKT, when possible, is performed in adult recipients weighing $<70 \mathrm{~kg}$, aged $>50$ years and with controlled hypertension.

Although only a small number of EBKT recipients were included in this study, the results show excellent outcomes comparable with LDKT. "In the future, we may split en bloc kidneys and transplant them into two recipients when the donor's weight is $>10 \mathrm{~kg}$, which will further help to alleviate the problem of organ shortage," says Sharma.

\section{Helene Myrvang}

Original article Sharma, A. et al. En bloc kidney transplantation from pediatric donors: comparable outcomes with living donor kidney transplantation. Transplantation http://dx.doi.org/10.1097/TP.0b013e3182279107 